\title{
Dispositivos de asistencia ventricular como tratamiento de destino. A propósito de un caso
}

Guillermo Careaga-Reyna, Hugo Jesús Zetina-Tun, José Galván-Díaz y Luis Manuel Álvarez-Sánchez

Instituto Mexicano del Seguro Social, Centro Médico Nacional La Raza, Hospital General "Dr. Gaudencio González Garza", Ciudad de México, México

\begin{abstract}
Resumen
El tratamiento de la falla cardiaca terminal avanzada ha sido el trasplante de corazón, sin embargo, hay limitantes en cuanto a las características del receptor y disponibilidad de donadores, por lo que se ha utilizado alternativamente el soporte ventricular como tratamiento de destino. Se describe a una paciente con falla cardiaca terminal por cardiomiopatía isquémica en quien se implantó exitosamente un sistema de soporte ventricular izquierdo y a ocho meses del procedimiento se encontraba en clase funcional I, sin complicaciones.
\end{abstract}

PALABRAS CLAVE: Falla cardiaca terminal. Soporte ventricular izquierdo. Terapia de destino. Trasplante cardiaco. Dispositivo de asistencia ventricular.

\begin{abstract}
The treatment of refractory terminal heart failure has been heart transplantation. However, there are limitations with regard to clinical conditions of the recipient and availability of donors, and ventricular support has therefore been alternatively used as destination therapy. We describe the case is of a female patient with ischemic cardiomyopathy-associated heart failure who had a left ventricular assist device successfully implanted and at eight months of the procedure was at functional class I, with no complications.
\end{abstract}

KEY WORDS: Terminal heart failure. Left ventricular support. Destination therapy. Heart transplantation. Ventricular assist device.

\section{Introducción}

La insuficiencia cardiaca terminal se ha incrementado en las últimas décadas debido a mejores tratamientos médicos y quirúrgicos que prolongan la vida, pero que no en todos los casos se evita la progresión del daño del miocardio. Indudablemente, el trasplante de corazón es el manejo definitivo de la insuficiencia cardiaca terminal, ${ }^{1}$ ya que logra una supervivencia de 10.7 a 13.6 años. ${ }^{2}$ Sin embargo, la principal limitante es la disponibilidad de donadores apropiados, pues se atiende menos de $10 \%$ de los casos con 2500 órganos ofertados, para una potencial demanda de 250000 pacientes que requieren un trasplante cardiaco. Por esta situación se buscó la opción de dispositivos de asistencia ventricular como alternativa terapéutica. En la actualidad hay series que indican que más de $40 \%$ de los pacientes en lista de espera de trasplante tienen implantado un sistema de apoyo ventricular. ${ }^{3}$

La expectativa de vida en México es de 75 años en el hombre y 77 años en la mujer. En 2002, la Food and Drug Administration de Estados Unidos aprobó el uso de dispositivos de asistencia ventricular (VAD, ventricular assist device) como puente al trasplante cardiaco en individuos que desarrollan falla terminal a pesar de terapia óptima con apoyo inotrópico o en quienes se considera que tendrán un tiempo de
Correspondencia:

Guillermo Careaga-Reyna

E-mail: gcareaga3@gmail.com
Fecha de recepción: 30-03-2018

Fecha de aceptación: 22-11-2018

DOI:10.24875/GMM.18004321
Gac Med Mex. 2019;155:196-198

Disponible en PubMed

www.gacetamedicademexico.com 
espera prolongado. ${ }^{3}$ A partir de 2010, los VAD fueron aprobados como tratamiento de destino (TD) para el manejo de la falla cardiaca terminal. Las indicaciones para su implante son muy precisas: puente a trasplante cardiaco, puente a otro proceso por determinar, TD, puente a recuperación, rescate, etcétera.

Debido a la escasez de injertos cardiacos disponibles, el implante de los VAD se ha incrementado en todo el mundo, sobre todo como TD. Los VAD son de duración temporal (<30 días) o prolongada ( $>30$ días a años); soportan y ayudan a la función cardiaca deteriorada, mejorando el estado clínico, funcional y calidad de vida del paciente.

Se presenta un caso en el que se utilizó un sistema de apoyo ventricular prolongado como TD.

\section{Presentación del caso}

Mujer de 68 años entre cuyos antecedentes de importancia destacaba dislipidemia en tratamiento con atorvastatina y bezafibrato. Tuvo infarto agudo del miocardio seis años atrás, sin tratamiento de reperfusión; egresó a las 48 horas por mejoría. Posteriormente presentó deterioro progresivo de la clase funcional; tres meses después se le colocó desfibrilador automático implantable en modo DD, con frecuencia máxima de 120 estímulos por minuto, frecuencia mínima de 70 , taquicardia ventricular de 171 y fibrilación ventricular de 214. A seis meses del implante se presentó la solicitud de trasplante al comité respectivo del hospital donde era atendida; aunque fue aceptada, ante la falta de donador apropiado y persistencia del deterioro clínico, angina a medianos esfuerzos, pérdida de peso de $15 \mathrm{~kg}$ y reingresos hospitalarios frecuentes por insuficiencia cardiaca en clases funcionales III a IV, se revaloró el caso y se optó por colocar un VAD izquierdo como TD. En la valoración preoperatoria se encontró colecistitis litiásica; se efectuó colecistectomía laparoscópica.

El ecocardiograma mostró válvula mitral con área de $4 \mathrm{~cm}^{2}$, velocidad máxima (Vmáx) de 1.9 , sin insuficiencia; válvula aórtica trivalva, con área de $2.22 \mathrm{~cm}^{2}$, Vmáx de 1.9 , gradiente máximo de $14 \mathrm{~mm} \mathrm{Hg}$, sin insuficiencia; válvula tricúspide con Vmáx de 2.9 e insuficiencia moderada; presión sistólica de la arteria pulmonar de $43 \mathrm{~mm} \mathrm{Hg}$, disfunción sistólica severa del ventrículo izquierdo con fracción de expulsión (FEVI $28 \%$ ), diámetro sistólico de $52 \mathrm{~mm}$ y diastólico de 64, con septum interventricular con grosor de $10 \mathrm{~mm}$. El gammagrama mostró infarto anteroseptal sin viabilidad miocárdica. Los hallazgos en el cateterismo cardiaco fueron tronco coronario izquierdo sin lesiones, descendente anterior,
Gensini 3, ectasia focal de los segmentos proximal y medio, el resto del vaso con diámetro menor a $2 \mathrm{~mm}$, primera diagonal sin lesiones, arteria circunfleja no dominante, sin lesiones, rama ventricular izquierda y descendente posterior sin lesiones. La presión del ventrículo izquierdo fue de $107 / 79 \mathrm{~mm} \mathrm{Hg}$, aorta de $110 / 72$ $\mathrm{mm} \mathrm{Hg}$, tronco de arteria pulmonar de $53 / 23 \mathrm{~mm} \mathrm{Hg}$, ventrículo derecho de $51 / 2 \mathrm{~mm} \mathrm{Hg}$, aurícula derecha de $2 \mathrm{~mm} \mathrm{Hg}$. Se repitió la toma de presiones derechas y los resultados fueron ventrículo derecho de $46 / 6 \mathrm{~mm} \mathrm{Hg}$, arteria pulmonar de $46 / 7 \mathrm{~mm} \mathrm{Hg}$ y presión capilar pulmonar de $4 \mathrm{~mm} \mathrm{Hg}$.

Sin contraindicaciones para cirugía y evaluación con la escala de la Sociedad de Cirujanos Torácicos, $8.2 \%$ de riesgo de mortalidad, $45 \%$ de morbilidad, $38 \%$ de falla renal y $25 \%$ de necesidad de reintervención, se procedió a implante de dispositivo de asistencia ventricular izquierda Heart-Mate II®.

El procedimiento se realizó bajo anestesia general balanceada, a través de esternotomía media longitudinal y con apoyo de circulación extracorpórea (Figuras 1 y 2). Consistió en crear una bolsa subdiafragmática para el sistema de bombeo e implantar a través de orificio en ápex del ventrículo izquierdo el tubo de salida de sangre, para pasar a través de la bomba en dirección a un injerto precoagulado que se anastomosó término-lateral a la aorta ascendente. El sistema se puso en funcionamiento, previa exteriorización del cable de alimentación de energía y conexión a la batería externa. Se retiró gradualmente la derivación cardiopulmonar y el sistema de apoyo se hizo cargo progresivamente de la función de bombeo. Se efectuó hemostasia cuidadosa y se colocaron drenajes torácicos para proceder al cierre de la pared torácica de la forma habitual. No hubo complicaciones posoperatorias y la paciente se mantuvo en terapia intensiva posquirúrgica por 10 días, al cabo de los cuales egresó a hospitalización, donde la familia y la paciente fueron nuevamente capacitados en el uso del sistema, el cambio de baterías, la forma de carga y las opciones de uso para que la paciente pudiera desarrollar sus actividades sin complicaciones. La paciente egresó del hospital 28 día después del implante. Al momento de este informe se encontraba en clase funcional I, había recuperado peso y a siete meses de la cirugía no presentaba complicaciones.

\section{Discusión}

El estudio INTERMACS es un registro de pacientes estadounidenses que recibieron apoyo ventricular 


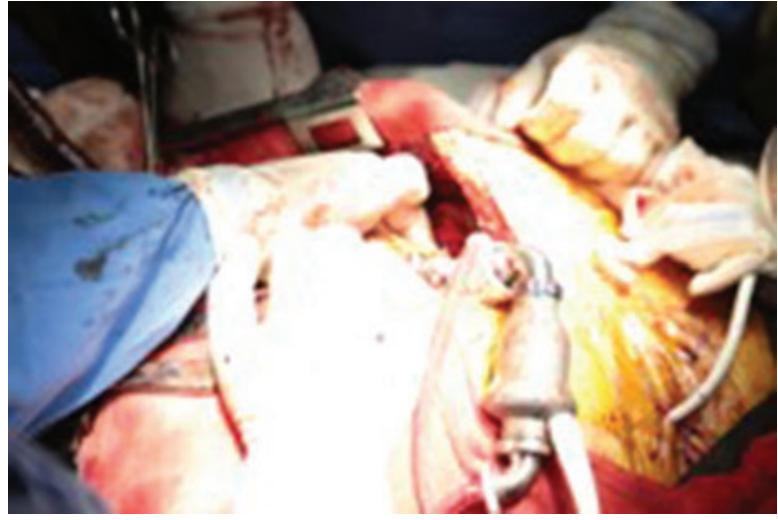

Figura 1. Implantación del dispositivo de asistencia ventricular en la bolsa subdiafragmática.

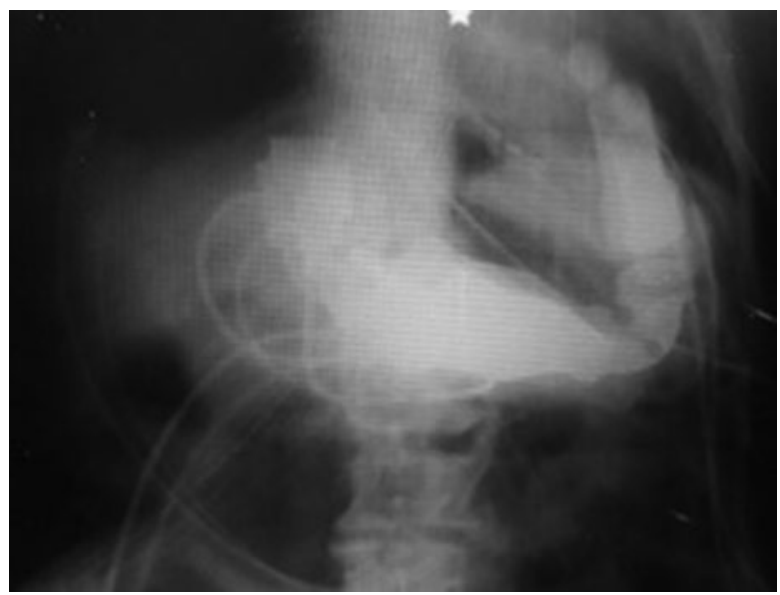

Figura 2. Control radiológico posoperatorio de la posición apropiada del dispositivo de asistencia ventricular.

mecánico para tratar la falla cardiaca terminal; de este estudio derivaron siete niveles de indicación de acuerdo con el perfil hemodinámico y capacidad funcional. ${ }^{4}$ Desde el estudio de REMARCH 2001, donde se utilizó el Heart Mate II, se constató que la instalación de VAD como TD derivó en mejor supervivencia a un año que solo el manejo con terapia médica.

El estudio ROADMAP ${ }^{5}$ comparó la instalación de VAD como TD versus tratamiento médico; si bien la mortalidad a un año fue similar (20\%), la calidad de vida y capacidad funcional mejoraron en quienes se implantó el VAD.

Según datos del ISHLT Mechanically Assisted Circulatory Support Registry Steering, hasta 2012 se habían implantado seis mil dispositivos en el mundo y la indicación principal fue el TD (40\%) seguido de puente a trasplante cardiaco (39\%); el sexo más frecuente es el masculino (78 \%) y el intervalo de edad en que se colocan con más frecuencia es entre $60 \mathrm{y}$ 79 años (45\%), seguido de 40 a 59 años (42\%).
El tipo de VAD más frecuentemente implantado es el tipo soporte ventricular izquierdo de tipo flujo axial (99\%); el Heart Mate II pertenece a este grupo. La tasa de supervivencia a 12 y 18 años es de 78 y $71 \%$, respectivamente. La supervivencia de los pacientes con VAD como puente a trasplante cardiaco a 12 y 18 meses fue de 85 y $82 \%$, con mejor pronóstico que los pacientes manejados solo con tratamiento médico.

Los VAD como TD ha mejorado la calidad de vida de los individuos mayores de 75 años. Un único estudio comparó VAD como TD y trasplante cardiaco en el grupo etario de 65 a 72 años, con resultados similares a dos años, si bien este recurso tendría mejor beneficio en individuos menores de 60 años.

El uso crónico del dispositivo no está libre de complicaciones, pues se ha observado que hasta $20 \%$ de los pacientes presenta infecciones del cable del control a dos años del implante, $10 \%$ eventos cerebrovasculares, mayor frecuencia (50\%) de episodios de epistaxis o sangrado gastrointestinal y menor frecuencia de trombosis del dispositivo; en $4 \%$ ha sido necesario cambiar el controlador. ${ }^{6}$ Los VAD han logrado disminuir la lista de receptores de trasplante cardiaco, a tal punto que se piensa que este último será una opción en un futuro para los pacientes portadores de VAD que se compliquen por infección, trombosis del dispositivo o mal funcionamiento del VAD, si bien el pronóstico de supervivencia disminuye si dichas complicaciones están presentes al momento de trasplante.

En cuanto al costo-beneficio, el VAD es más caro que el trasplante cardiaco con complicaciones en los dos años siguientes: infecciones (19\%), sepsis (19\%), eventos vasculares cerebrales (12\%), hemorragias (54\%) o falla ventricular derecha (18\%).

\section{Bibliografía}

1. Careaga-Reyna G, Zetina-Tun H, Lezama-Urtecho CA. Programa de trasplante cardiaco de la Unidad Médica de Alta Especialidad, Hospital General Dr. Gaudencio González Garza del Centro Médico Nacional "La Raza". Rev Invest Clin. 2011;63:85-90.

2. Stehlik J, Edwards LB, Kucheryavaya AY, Benden C, Christie JD, Dobbels $F$, et al. The Registry of the International Society for Heart and Lung Transplantation: twenty-eighth adult heart transplant report-2011. J Heart Lung Transplant. 2011;30:1078-1094.

3. Mancini D, Colombo PC. Left ventricular assist devices: a rapidly evolving alternative to transplant. J Am Coll Cardiol. 2015;65:2542-2555.

4. Kirklin JK, Naftel DC, Kormos RL, Pagani FD, Myers SL, Stevenson LW, et al. Interagency Registry for Mechanically Assisted Circulatory Support (INTERMACS) analysis of pump thrombosis in the HeartMate II left ventricular assist device. J Heart Lung Transplant. 2014;33:12-22.

5. Estep JD, Starling RC, Horstmanshof DA, Milano CA, Selzman $\mathrm{CH}$, Shah KB, et al. Risk assessment and comparative effectiveness of left ventricular assist device and medical management in ambulatory heart failure patients: results from the ROADMAP. J Am Coll Cardiol. 2015;66:1747-1761.

6. Echeverría L, Salazar L, Torres A, Figueredo A. Dispositivos de asistencia ventricular: una realidad en Colombia. Rev Colomb Cardiol. 2016;23:49-54. 